\title{
Development of Variable Rate Sprayer for Oil Palm Plantation.
}

\begin{abstract}
This project describes the design and development of a camera vision with color detection for the variable rate technology (VRT) automated sprayer. In this project, the smart sprayer system was already developed and mounted on the ATV. The automated sprayer system was developed by combination of the electromechanical system, PC parallel port relay board, the controller and visual basic programming software. This smart sprayer system is guided with the camera to detect the presence of weeds. Detection of weeds is based on the green color value from RGB value. The amount or percentage $(\%)$ of weeds detected determines the rate of spraying that is controlled by an electric motor and the relay board. In this project, the spray nozzles were modified to be fully open, fully closed and half open. The closing and opening of valves were controlled by the electromechanical system that receives the instruction from the camera vision. Experiments carried out shows that the nozzle is closed when the percentage of weeds detected is less than $2 \%$. It is half open at $3 \%$ to $50 \%$ and fully open at more than $51 \%$. The application rate of spraying can be determined from the result of the spraying operation.
\end{abstract}

Keyword: Chemical sprayer; Boom sprayer; Camera; RGB; Computer pixel; Normally open valve; Nnormally closed valve. 